\title{
Correction to: Tat-NTS Suppresses the Proliferation, Migration and Invasion of Glioblastoma Cells by Inhibiting Annexin-A1 Nuclear Translocation
}

\author{
Zhenzhao Luo ${ }^{1} \cdot \mathrm{Li} \mathrm{Liu}^{2} \cdot \mathrm{Xing} \mathrm{Li}^{3} \cdot$ Weiqun Chen ${ }^{4} \cdot$ Zhongxin Lu$^{1} \mathbb{C}$
}

Published online: 3 September 2021

๑) Springer Science+Business Media, LLC, part of Springer Nature 2021

\section{Correction to: Cellular and Molecular Neurobiology https://doi.org/10.1007/s10571-021-01134-y}

The original version of this article unfortunately contained error in figure legends.

The article was published with incomplete figure legends for all the figures. Hence, the complete figure legends with its corresponding Figs. 1, 2, 3, 4 and 5 are presented here.

The original article has been corrected.

Publisher's Note Springer Nature remains neutral with regard to jurisdictional claims in published maps and institutional affiliations.

The original article can be found online at https://doi.org/10.1007/ s10571-021-01134-y.

\section{Zhongxin Lu}

lzx71@yahoo.com.cn

1 Department of Medical Laboratory, The Central Hospital of Wuhan, Tongji Medical College, Huazhong University of Science and Technology, 26 Shengli St., Jiangan District, Wuhan 430014, China

2 Department of Respiration, The Children's Hospital of Wuhan, Tongji Medical College, Huazhong University of Science and Technology, Wuhan 430015, China

3 Department of Neurobiology, The School of Basic Medical Science, Tongji Medical College, Huazhong University of Science and Technology, Wuhan 430030, China

4 Key Laboratory for Molecular Diagnosis of Hubei Province, The Central Hospital of Wuhan, Tongji Medical College, Huazhong University of Science and Technology, Wuhan 430014, China 
Fig. 1 Effect of Tat-NTS on GB cell viability. Human U87 and U251 GB cells and normal human astrocytes (NHAs) were exposed to various concentrations of Tat-NTS $(2,5,10,20$ and $40 \mu \mathrm{M}$ ) or PBS (vehicle) for $24 \mathrm{~h}$. Cells were also exposed to $20 \mu \mathrm{M}$ Tat-NTS for 12,24 and $48 \mathrm{~h}$. Cell viability was then analyzed by CCK- 8 assay. Tat-NTS inhibited GB cell proliferation in a dose-dependent manner (a) and time-dependent manner (b) but had no impact on NHAs. Mean \pm SD $(n=5)$. $* P<0.05$ and $* * P<0.01$ vs $0 \mu \mathrm{mol} / \mathrm{L}$ or $0 \mathrm{~h}$. c Expression of the proliferation marker Ki-67 was evaluated using immunofluorescence staining for Ki67 (red) and DAPI (blue). Scale bar, $40 \mu \mathrm{m}$

\section{A}

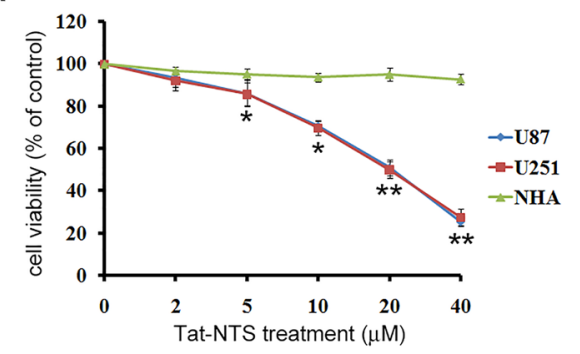

C

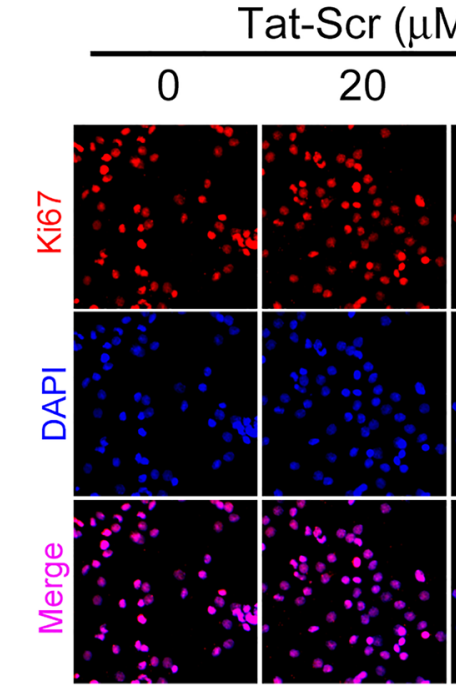

B

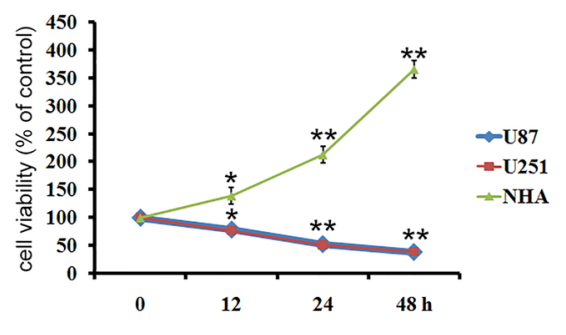

Tat-NTS $(\mu \mathrm{M})$
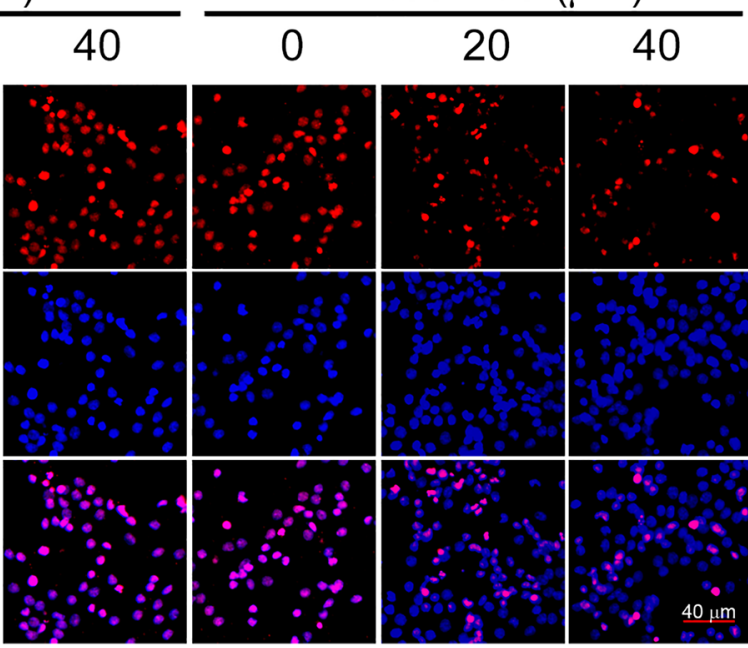

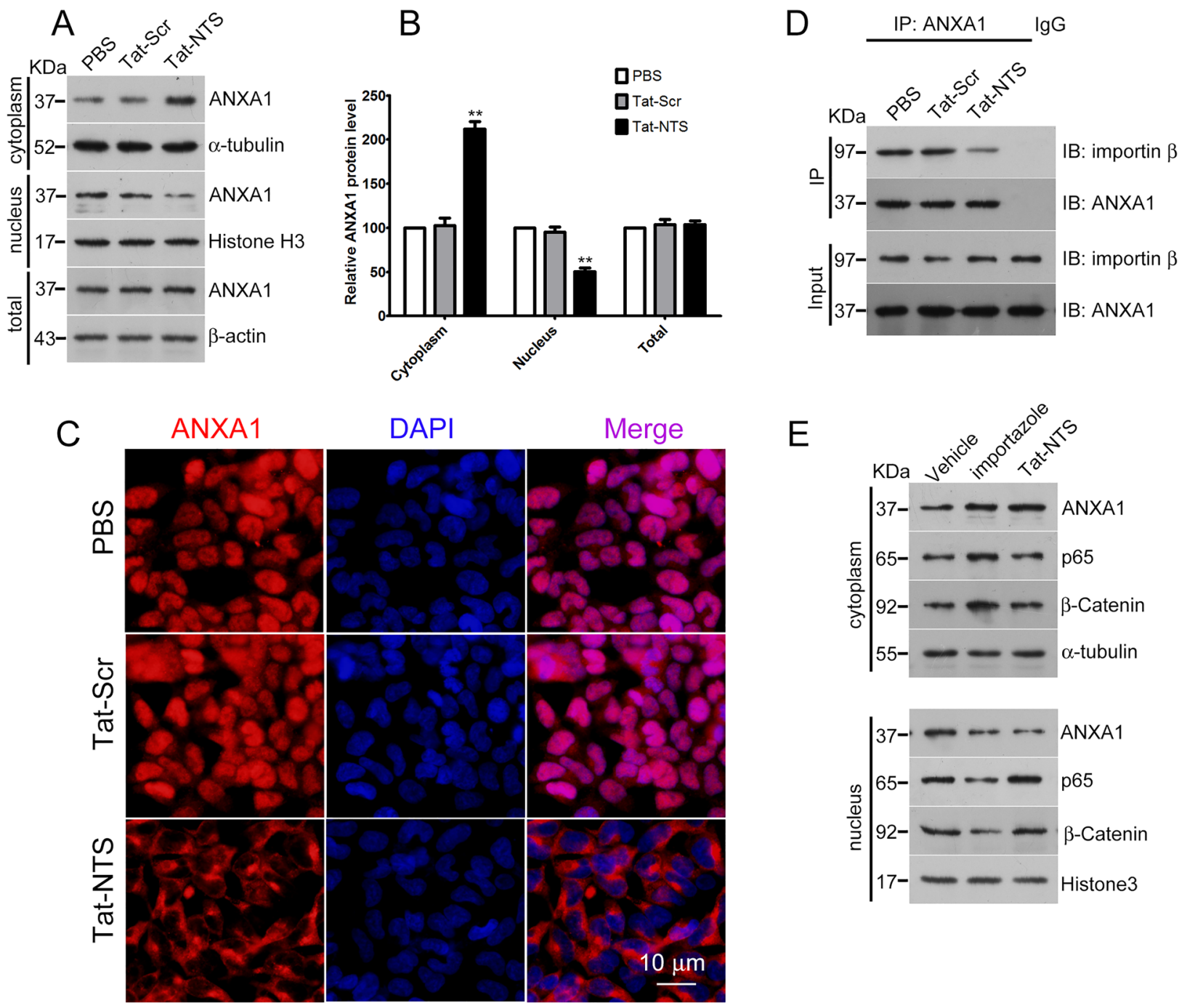

$E$
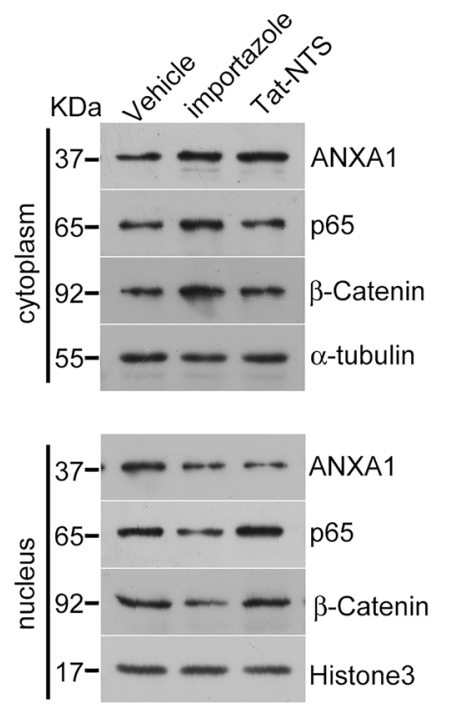

$\mathrm{F}$

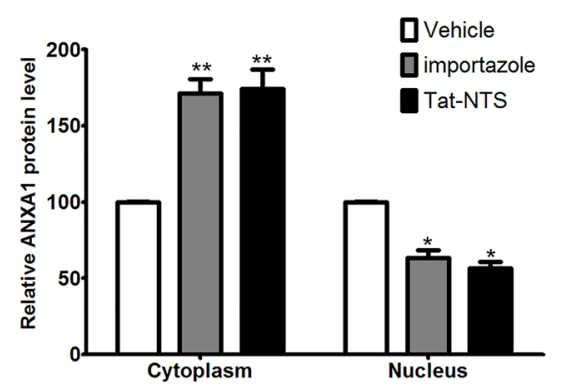

G

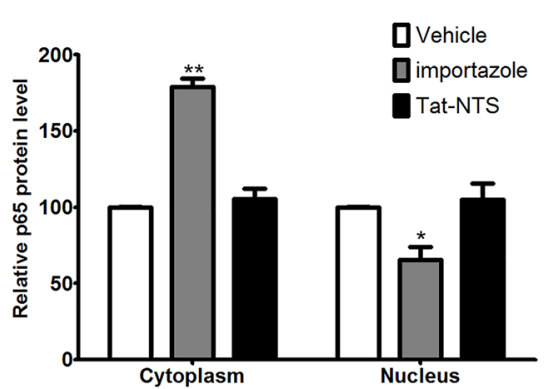

$\mathrm{H}$

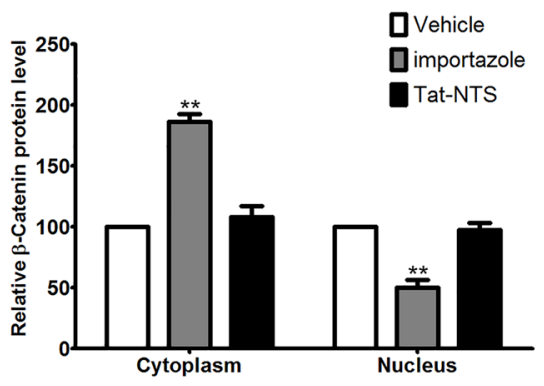

Fig. 2 Tat-NTS inhibits ANXA1 translocation to the nucleus in U87 cells. U87 cells were treated with PBS, Tat-Scr or Tat-NTS $(20 \mu \mathrm{M})$ for $24 \mathrm{~h}$. Nuclear and cytosolic protein extracts were then subjected to Western blot analysis with specific antibodies. a Representative images of Western blot analysis for ANXA1 levels in the cytoplasmic and nuclear extracts. $\alpha$-tubulin and histone $\mathrm{H} 3$ were used as cytoplasmic and nuclear internal controls, respectively, while $\beta$-actin was used as a loading control of total proteins. b The relative level of ANXA1 protein was quantified by densitometric analysis. Data are represented as the mean \pm SD from three independent experiments. $* * P<0.01$ vs the Tat-Scr control. c Representative immunofluorescence staining results of ANXA1 (red) and nuclei (blue) showing the subcellular distribution of endogenous ANXA1 in U87 cells. Scale bar $=10 \mu \mathrm{m}$. d Representative Co-IP results showing the interaction of ANXA1 with importin $\beta$ in GB cells treated with Tat-NTS or Tat-Scr peptide. e Representative images following Western blot analysis for ANXA1, p65 NF- $\kappa \mathrm{B}$, and $\beta$-catenin in cytoplasmic and nuclear extracts of U87 cells treated with vehicle, importazole or TatNTS peptide. $\alpha$-Tubulin and histone $\mathrm{H} 3$ served as cytoplasmic and nuclear loading controls, respectively. $\mathbf{f}-\mathbf{h}$ Quantification of the relative protein levels of ANXA1, p65 NF- $\mathrm{KB}$ and $\beta$-catenin. The intensity of the bands was quantified by densitometric analysis. $* P<0.05$ and $* * P<0.01$ vs the Vehicle group; all data are presented as the mean \pm SD from three independent experiments 
Fig. 3 Effect of Tat-NTS on the migration and invasion capacity of GB cells in vitro. a-c U87 GB cells, U251 GB cells and NHAs were treated with PBS, Tat-Scr $(20 \mu \mathrm{M})$ or Tat-NTS $(20 \mu \mathrm{M})$ for $24 \mathrm{~h}$, and cell migration was then determined with a Transwell system. Migrated and invaded cells on the bottom surface of the membrane were fixed and stained with crystal violet.

Original magnification: $\times 200$. d-f Quantification of migrating and invading cells corresponding to $\mathbf{a}-\mathbf{c}$. The migrating and invading cells were counted under a microscope in five random fields. Data are presented as the mean \pm SD from three independent experiments. $* * P<0.01$ vs the Tat-Src group

A

PBS Tat-Scr

Tat-NTS

U87

B

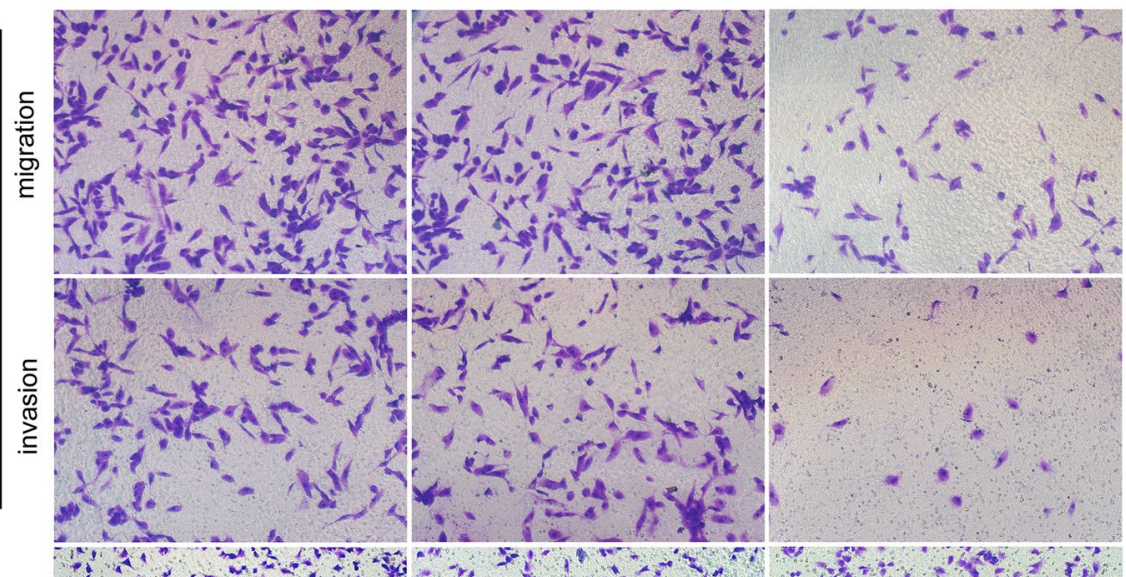

U251

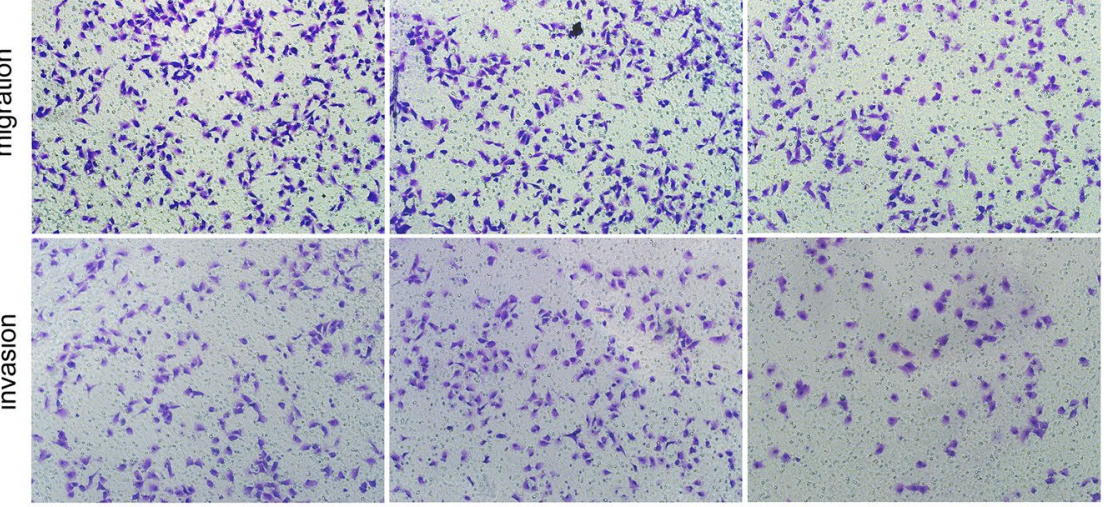

C

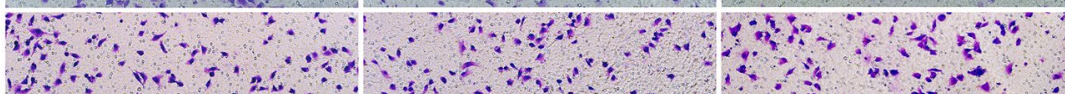

$\mathrm{NHA}$

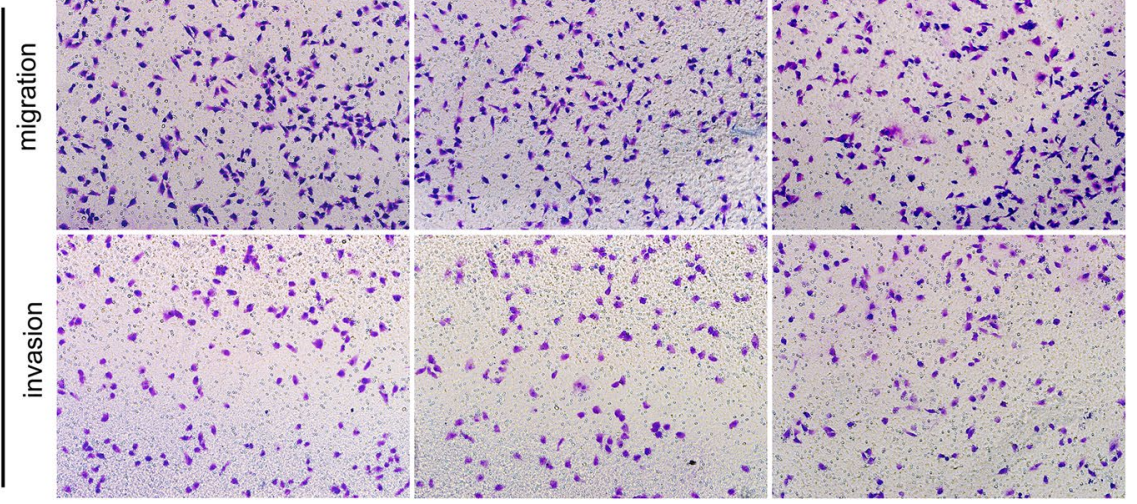

D

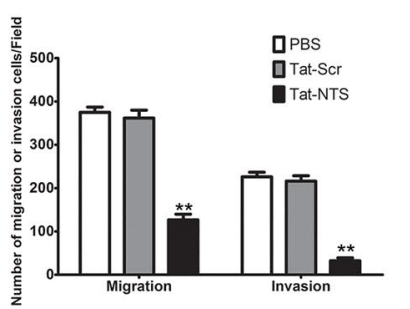

E

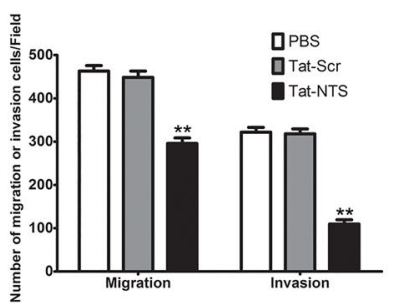

F

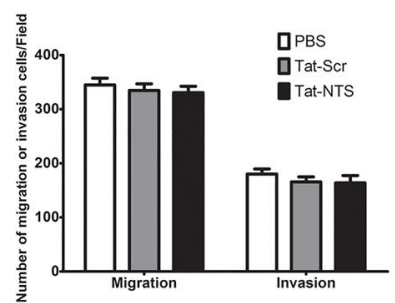


A

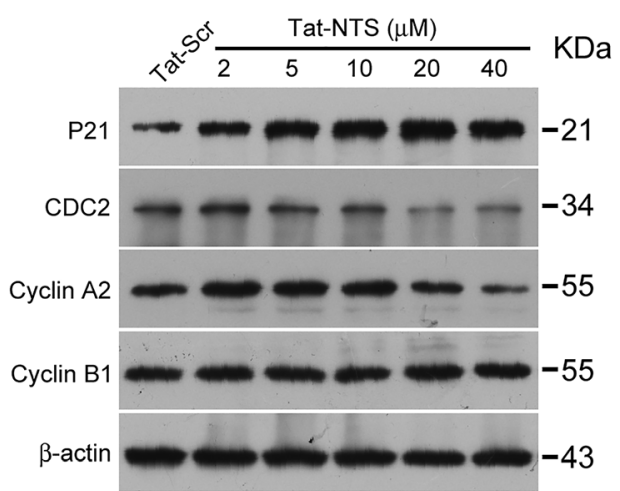

C

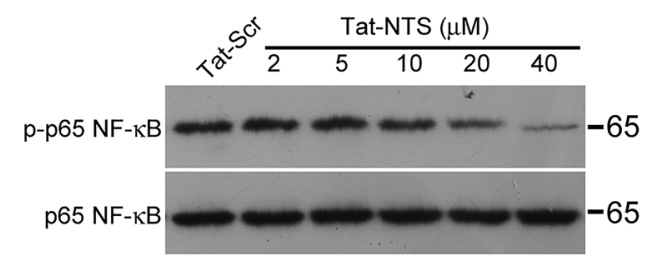

B

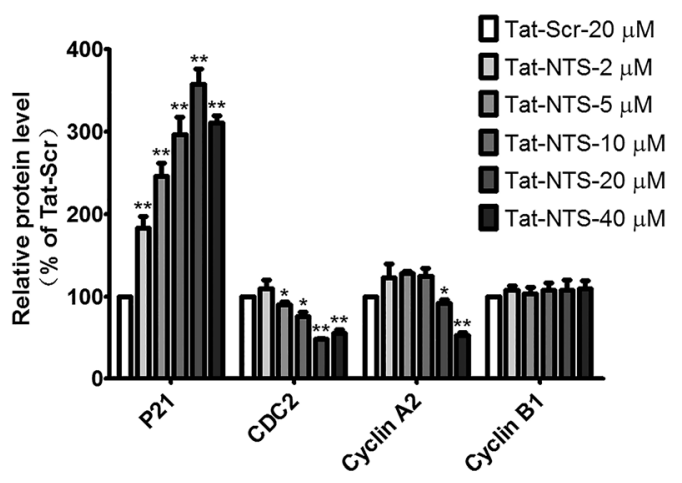

D

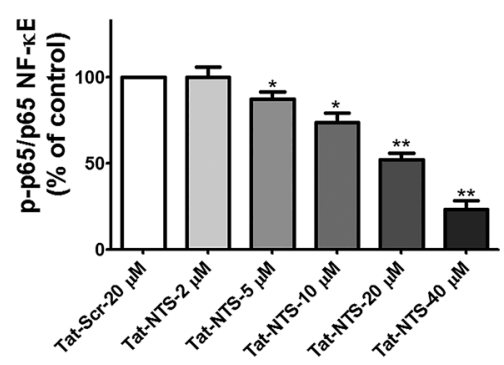

determined by Western blot analysis. a and $\mathbf{c}$ Representative protein blots evaluated by Western blot analysis. $\mathbf{b}$ and $\mathbf{d}$ Densities of the protein bands were normalized to those of $\beta$-actin and p65 NF- $\kappa$ B. Data are shown as the mean \pm SD from three independent experiments. $* P<0.05$ and $* * P<0.01$ vs the Tat-Scr group 


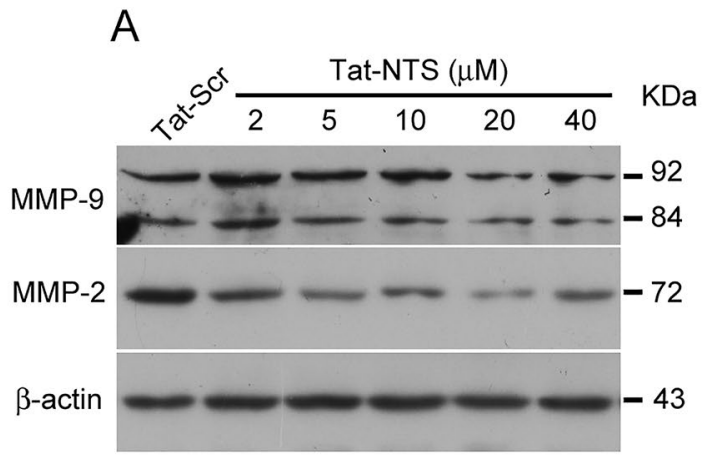

C

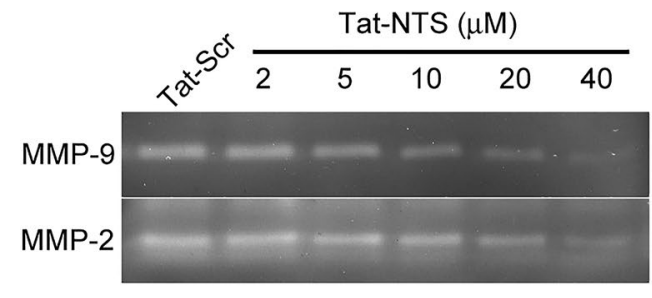

B
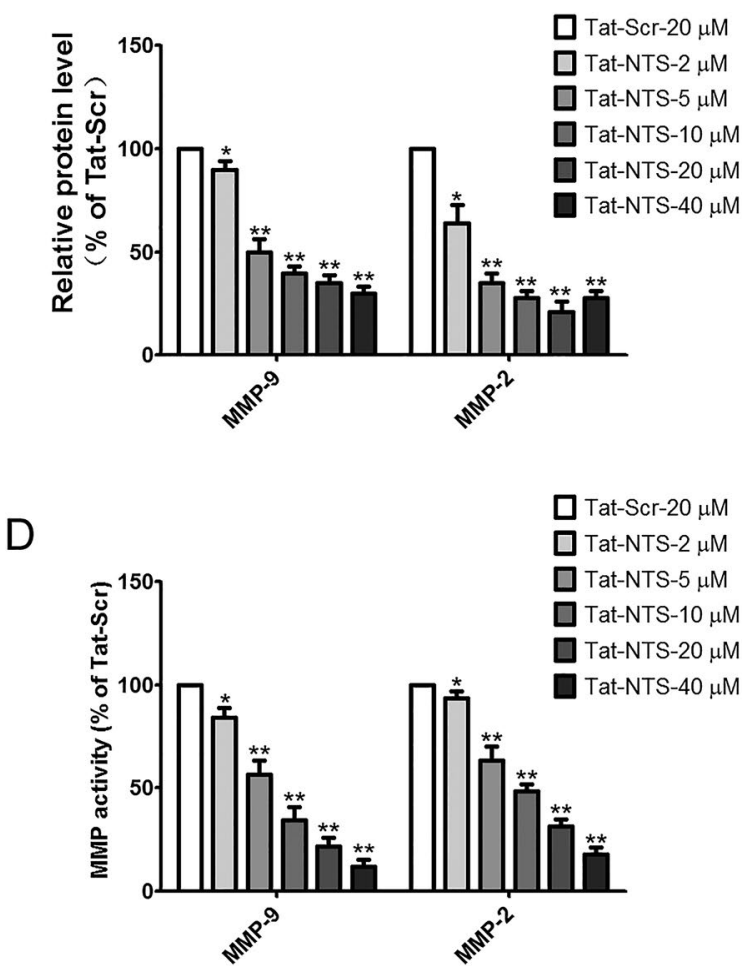

lowing Western blot analysis for MMP-2 and MMP-9 expression in U87 cells. b Quantification of relative protein levels of MMP-2 and MMP-9 in U87 cells. c Representative images of gelatin zymography of MMP-2 and MMP-9. d Quantification of the relative bands of MMP-2 and MMP-9 in U87 cells $(n=3) . * P<0.05$ and $* * P<0.01$ vs the Tat-Scr group 